\title{
Transient splenial lesion of the corpus callosum in a case of benign convulsion associated with rotaviral gastroenteritis
}

Yoon Young Jang, M.D. and Kye Hyang Lee, M.D.

Department of Pediatrics, College of Medicine, Daegu Catholic University, Daegu, Korea
Received: 16 April 2010, Revised: 22 June, 2010 Accepted: 20 July 2010

Corresponding author: Kye Hyang Lee, M.D.

Department of Pediatrics, College of Medicine, Daegu Catholic University, 3056-6, Daemyeong 4-dong, Nam-gu, Daegu 705-718, Korea

Tel: +82.53-650-4242, Fax: +82.53-622-4240

E-mail: rosalia@cu.ac.kr

Copyright $(2010$ by The Korean Pediatric Society
Transient magnetic resonance (MR) signal changes in the splenium of the corpus callosum (SCC) arise from many different conditions, including encephalopathy or encephalitis caused by infection, seizures, metabolic derangements, and asphyxia. Few case reports exist on reversible SCC lesions associated with rotavirus infection. A benign convulsion with mild gastroenteritis ( $\mathrm{CwG}$ ) is frequently associated with rotaviral infections. This entity is characterized by normal laboratory findings, electroencephalogram, neuroimaging, and good prognosis. We report a case of a 2.5-year-old Korean girl with rotavirus-associated CWG demonstrating a reversible SCC lesion on diffusion-weighted MR images. She developed 2 episodes of brief generalized tonic-clonic seizure with mild acute gastroenteritis without any other neurologic abnormality. Stool test for rotavirus antigen was positive. Brain MRI done on the day of admission showed a linear high signal intensity and decreased apparent diffusion coefficient values on the SCC. The lesion completely disappeared on follow-up MRI 6 days later. The patient fully recovered without any sequelae.

Key words: Corpus callosum, Magnetic resonance imaging, Diffusion, Rotavirus infection, Seizures, Child

This is an open-access article distributed under the terms of the Creative Commons Attribution Non-Commercial License (http://creativecommons.org/licenses/by$\mathrm{nc} / 3.0 /$ ) which permits unrestricted non-commercial use, distribution, and reproduction in any medium, provided the original work is properly cited.

\section{Introduction}

Rotavirus is a major pathogen for viral gastroenteritis in infants and young children. It can be associated with central nervous system involvement, to include seizure with or without fever, aseptic meningitis, encephalopathy, encephalitis, and cerebellitis ${ }^{11}$. Benign convulsion with mild gastroenteritis $(\mathrm{CwG})$ is a common condition characterized by repetitive brief seizures associated with symptoms of gastroenteritis in infant and young children. Rotavirus antigen in stool has been detected in the majority of patients with CwG (more than $50 \%)^{2)}$. A transient lesion in the splenium of the corpus callosum (SCC) associated with rotaviral infection have been reported in several cases, most of them in patients with encephalopathy or encephalitis ${ }^{3,4}$. Few cases with rotavirus related CwG has shown a reversible SCC lesion ${ }^{5,6}$. There have been no case reports to date of transient SCC lesions associated with rotaviral infections in Korea. We report the first Korean case of a rotavirusassociated $\mathrm{CwG}$ demonstrating a transient SCC lesion. 


\section{Case report}

A 2.5-year-old girl was admitted for two episodes of generalized tonic-clonic seizures. She presented with a 2 day history of vomiting, diarrhea and fever. On the day of admission, sudden onset of generalized tonic-clonic seizures lasting 30 seconds developed while crying. Prior to transfer to our hospital, the patient reportedly developed another generalized tonic-clonic seizure lasting 1 minute while at a local hospital emergency department. Her past medical history was unremarkable with normal development except for one febrile convulsion episode at 13 months of age. Family history was also unremarkable upon review. On physical examination, she was alert and her body temperature was $37.8^{\circ} \mathrm{C}$. The oral mucosa and lips were not dry and skin turgor was fair. The abdomen was mildly distended with slight-hyperactive bowel sounds. Neurologic examination revealed no abnormality. Laboratory findings included a leukocyte count of 5,600/ $\mathrm{L}$, hemoglobin $12.4 \mathrm{~g} / \mathrm{dL}$, platelet count $227,000 / \mu \mathrm{L}$, serum sodium $138 \mathrm{mEq} / \mathrm{L}$, potassium $3.6 \mathrm{mEq} / \mathrm{L}$, chlorine $108 \mathrm{mEq} / \mathrm{L}$, bicarbonate $17 \mathrm{mmoL} / \mathrm{L}, \mathrm{BUN}$ $8.0 \mathrm{mg} / \mathrm{dL}$, creatinine $0.6 \mathrm{mg} / \mathrm{dL}$, glucose $83 \mathrm{mg} / \mathrm{dL}$, ammonia 21 $\mu \mathrm{mol} / \mathrm{L}$, and calcium of $9.1 \mathrm{mg} / \mathrm{dL}$. Cerebrospinal fluid analysis was not performed due to parental refusal for consent. Rotavirus stool antigen was positive. Electroencephalography (EEG) was normal. Brain MRI was done on admission day with T1-weighted, T2-weighted, and fluid-attenuated inversion recovery (FLAIR) images not revealing any abnormal findings. However, diffusionweighted images (DWI) showed linear high signal intensity in the splenium of the corpus callosum with decreased apparent

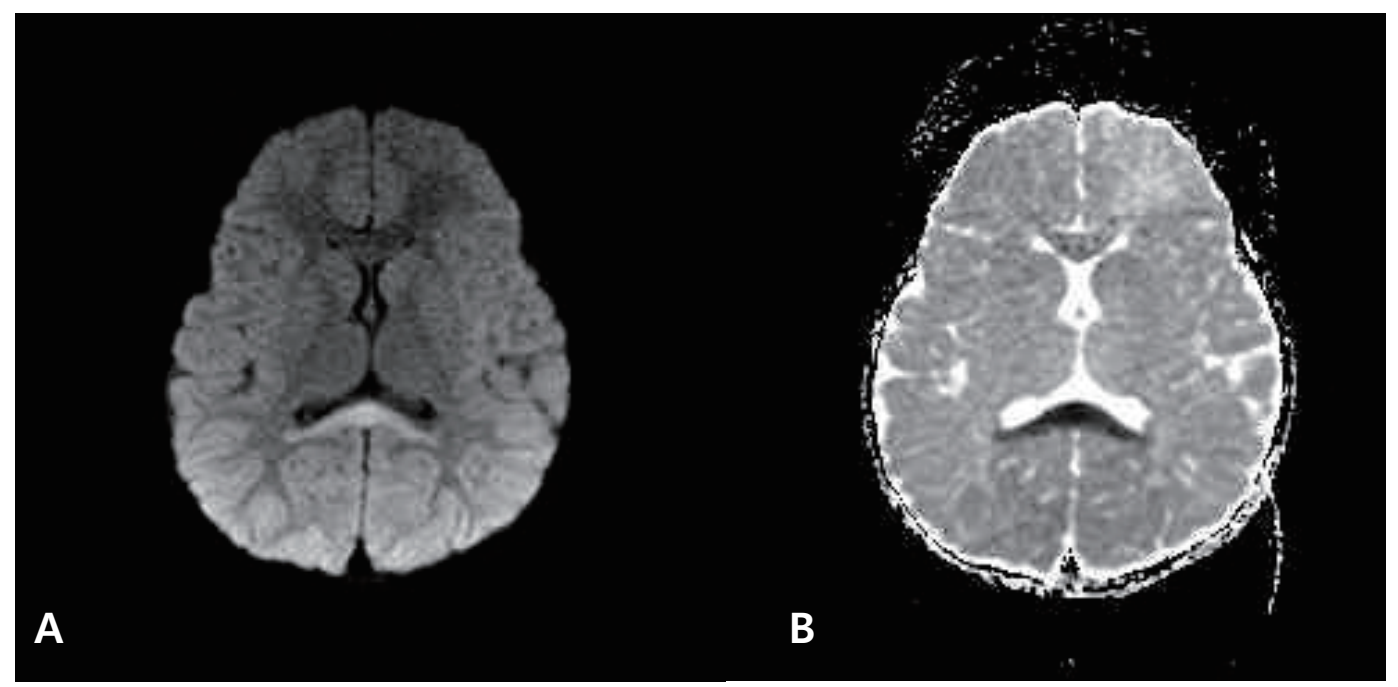

Fig. 1. Axial diffusion-weighted magnetic resonance images (A) and apparent diffusion coefficient map (B) demonstrate linear high signal intensities on the splenium of the corpus callosum on the day of admission.

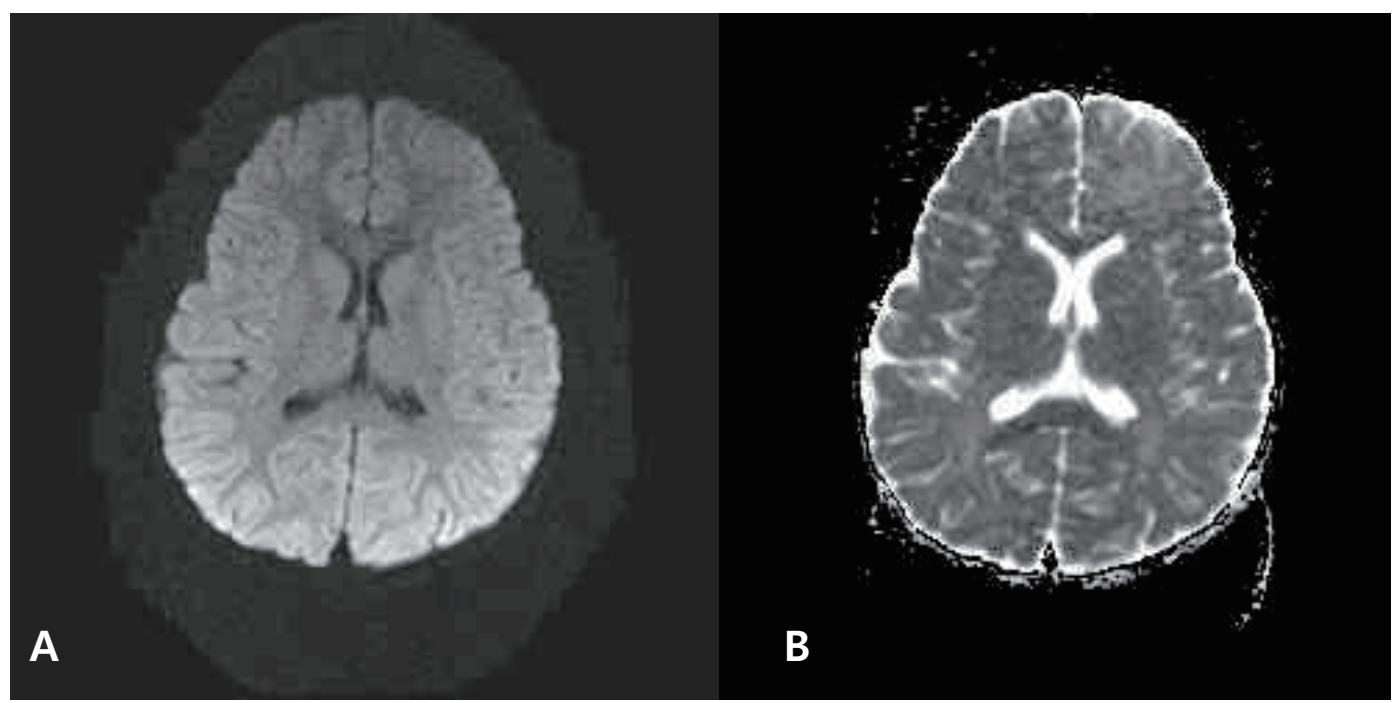

Fig. 2. Axial diffusion-weighted magnetic resonance images (A) and apparent diffusion coefficient map (B) demonstrate complete disappearance of the lesion on the splenium of the corpus callosum on the 6th day of admission. 
diffusion coefficient (ADC) values on the same area, suggesting cytotoxic edema (Fig. 1). The patient was treated with intravenous crystalloids, empiric antibiotics and acyclovir under initial presumptive diagnosis of meningoencephalitis. No anticonvulsants were administered. During the patient's admission, no additional convulsions or new neurologic abnormalities were noted. Mild symptoms of gastroenteritis persisted during her hospital course. A follow-up brain DWI MRI on the sixth day after admission revealed complete disappearance of the original lesion (Fig.2). The patient recovered completely without neurologic complications and was discharged after a 1 week hospital stay.

\section{Discussion}

Benign convulsions associated with mild, gastroenteritis or afebrile benign convulsions with mild gastroenteritis characteristically occur in previously healthy young infants and children aged 6 months to 3 years. Patients are typically afebrile, presenting with brief generalized tonic-clonic convulsions between the first and fifth days of a mild gastroenteritis. Only a mild degree of dehydration (less than 5\%) is usually apparent and seizures are brief and tend to occur repetitively over several days. Interictal EEG do not typically show any epileptic discharges. Lastly, basic laboratory examinations, including cerebrospinal fluid, serum electrolytes, and blood glucose are usually unremarkable, with patients having a full recovery with good prognosis. The known etiologic infectious agents causing this gastroenteritis include the rotavirus, norovirus, and other small round viruses ${ }^{7}$. Neuroimgaing usually reveals no abnormalities, but recently there have been some cases reporting a transient abnormal SCC lesion on diffusion-weighted brain $\mathrm{MRI}^{5,6)}$. The differential diagnosis for lesions involving the SCC include ischemia, infection, posterior reversible encephalopathy syndrome, diffuse axonal injury, multiple sclerosis, hydrocephalus, Marchiafava-Bignami disease, adrenoleukodystrophy, AIDSdementia complex, lymphoma, epilepsy, and antiepileptic drug usage $^{8)}$. Transient signal changes in the SCC have been reported in many different conditions, including infectious encephalitis or encephalopathy by various pathogens (rotavirus, measles, herpesvirus 6, influenza, Salmonella organism, mumps, varicellazoster virus, adenovirus, O-157 Escherichia coli, Legionnaire's disease), those with or without epilepsy receiving antiepileptic drugs (AED), alcoholism, malnutrition, hypoglycemia, hypernatremia, trauma, asphyxia, acute encephalopathy associated with intravenous immunoglobulin therapy, and neoplasms ${ }^{8-10)}$. There are some reports demonstrating a transient splenial lesion in cases with rotavirus-associated encephalopathy. Those cases had clinical features of encephalopathy, such as disturbance of consciousness, agitation, irritability, confusion, disorientation as well as seizures ${ }^{3,}$ ${ }^{4)}$. A review by Kato et al. ${ }^{6}$ described 9 Japanese cases of transient SCC lesions occurring during a rotavirus infection. Among them, $89 \%(8 / 9)$ had encephalopathic patterns such as alterations in consciousness, abnormal behavior, while 1 had clinical features consistent with CwG. Transient SCC lesions are rarely seen in cases with rotavirus-associated CwG. Natsume et al. ${ }^{5)}$ reported 2 cases of transient splenial lesions in children with benign convulsions with gastroenteritis. Both cases had multiple seizures associated with acute gastroenteritis (one with a rotaviral infection) without other neurologic problems. Since lumbar punctures were not performed in those cases, encephalitis could not be completely ruled out. Our case had no other neurologic abnormalities except seizure activity in the setting of a normal EEG. Although cerebrospinal fluid was not analyzed and the possibility of rotavirus encephalitis can not be excluded entirely, our case is most consistent with benign convulsions associated with rotaviral gastroenteritis based on clinical history and findings. Since a transient SCC lesion results from many different conditions, the exact mechanisms explaining the findings are unknown and diverse. Tada et al." proposed two possible mechanisms for this lesion in encephalopathy/encephalitis patients. They proposed that SCC lesions may be secondary to intramyelinic edema from separation of myelin layers, or that an influx of inflammatory cells and macromolecules, combined with cytotoxic edema causes decreased ADC levels. This may help to explain the decrease of ADC in the splenium. Splenial lesions after seizures have been reported in patients with epilepsy. Splenial abnormality in epilepsy is considered to be multifactorial, with contributing factors to include seizure activity propagation, antiepileptic drugs or hormonal imbalance. Seizures impair glucose availability, leading to brief reversible failure of cellular fluid regulation at the splenium. Reduced ADC values and normalization on additional imaging suggest transient intracellular edema with diffusion restriction on the $\mathrm{SCC}^{8,10,11)}$. Oster et al. ${ }^{11)}$ reported two bitemporal lobe epilepsy patients showing a transient SCC lesion, a structure involved in seizure propagation not seizure initiation. They suggested that the possible mechanism involved a transient disturbance of energy metabolism and ionic transport resulting in reversible myelin vacuolization and intramyelin edema due to excessive repetitive activity of the commissural projection from the temporal structure in these patients. Recently Gröppel et al. ${ }^{12)}$ analyzed 24 epilepsy cases with a focal lesion in the SCC and evaluated the relationship between the lesion and possible etiologic factors: epilepsy syndrome, seizure types, frequency, localization of interictal spikes and ictal EEG patterns, antiepileptic drugs, 
and drug levels. However, they could not find a single etiologic factor responsible for the SCC lesion. The reversible SCC lesion could result from multiple conditions including rotaviral infection, seizures, $\mathrm{CwG}$, encephalitis, and encephalopathy. Considering that our case had many potential contributing factors for the lesion's appearance, controversy remains regarding the most probable diagnosis and mechanisms involved. Although the diagnosis of encephalitis remains a possibility, our patient had only 2 brief generalized seizures without any encephalopathic features during a rotaviral infection. Therefore, our case should be classified as a benign convulsion associated with a rotaviral gastroenteritis.

\section{References}

1) Dickey M, Jamison L, Michaud L, Care M, Bernstein DI, Staat MA. Rotavirus meningoencephalitis in a previously healthy child and a review of the literature. Pediatr Infect Dis J 2009;28:318-21.

2) Verrotti A, Tocco AM, Coppola GG, Altobelli E, Chiarelli F. Afebrile benign convulsions with mild gastroenteritis: a new entity? Acta Neurol Scand 2009;120:73-9.

3) Kobata R, Tsukahara H, Nakai A, Tanizawa A, Ishimori Y, Kawamura Y, et al. Transient MR signal changes in the splenium of the corpus callosum in rotavirus encephalopathy: value of diffusion-weighted imaging. J Comput Assist Tomogr 2002;26:825-8.
4) Fukuda S, Kishi K, Yasuda K, Sejima H, Yamaguchi S. Rotavirusassociated encephalopathy with a reversible splenial lesion. Pediatr Neurol 2009;40:131-3.

5) Natsume J, Naiki M, Yokotsuka T, Sofue A, Ikuta T, Kondo Y, et al. Transient splenial lesions in children with "benign convulsions with gastroenteritis". Brain Dev 2007;29:519-21.

6) Kato Z, Orii KE, Morimoto M, Sasai H, Funato M, Sawatari S, et al. A transient lesion in the corpus callosum during rotavirus infection. Pediatr Neurol 2009;41:467-9.

7) Kawano G, Oshige K, Syutou S, Koteda Y, Yokoyama T, Kim BG, et al. Benign infantile convulsions associated with mild gastroenteritis: a retrospective study of 39 cases including virological tests and efficacy of anticonvulsants. Brain Dev 2007;29:617-22.

8) Maeda M, Tsukahara H, Terada H, Nakaji S, Nakamura H, Oba H, et al. Reversible splenial lesion with restricted diffusion in a wide spectrum of diseases and conditions. J Neuroradiol 2006;33:229-36.

9) Tada H, Takanashi J, Barkovich AJ, Oba H, Maeda M, Tsukahara H, et al. Clinically mild encephalitis/encephalopathy with a reversible splenial lesion. Neurology 2004;63:1854-8.

10) Bulakbasi N, Kocaoglu M, Tayfun C, Ucoz T. Transient splenial lesion of the corpus callosum in clinically mild influenza-associated encephalitis/ encephalopathy. AJNR Am J Neuroradiol 2006;27:1983-6.

11) Oster J, Doherty C, Grant PE, Simon M, Cole AJ. Diffusion-weighted imaging abnormalities in the splenium after seizures. Epilepsia 2003; 44:852-4.

12) Gröppel G, Gallmetzer P, Prayer D, Serles W, Baumgartner C. Focal lesions in the splenium of the corpus callosum in patients with epilepsy. Epilepsia 2009;50:1354-60. 